\title{
Are Yawns really Contagious? A Critique and Quantification of Yawn Contagion
}

\author{
Rohan Kapitány $^{1,2} \cdot$ Mark Nielsen $^{1}$
}

Received: 3 October 2016 / Revised: 5 January 2017 / Accepted: 6 January 2017 /

Published online: 13 February 2017

C The Author(s) 2017. This article is published with open access at Springerlink.com

\begin{abstract}
Many diverse species yawn, suggesting ancient evolutionary roots. While yawning is widespread, the observation of contagious yawning is most often limited to apes and other mammals with sophisticated social cognition. This has led to speculation on the adaptive value of contagious yawning. Among this speculation are empirical and methodological assumptions demanding re-examination. In this paper we demonstrate that if yawns are not contagious, they may still appear to be so by way of a perceptual pattern-recognition error. Under a variety of conditions (including the assumption that yawns are contagious) we quantify (via models) the extent to which the empirical literature commits Type-1 error (i.e., incorrectly calling a spontaneous yawn 'contagious'). We report the results of a preregistered behavioural experiment to validate our model and support our criticisms. Finally, we quantify - based on a synthesis of behavioural and simulated data how 'contagious' a yawn is by describing the size of the influence a 'trigger' yawn has on the likelihood of a consequent yawn. We conclude by raising a number of empirical and methodological concerns that aid in resolving higher-order questions regarding the nature of contagious yawning, and make public our model to help aid further study and understanding.
\end{abstract}

Keywords Yawn · Contagious yawn · Agent-based model - Type-1 error - Cognitive error

Electronic supplementary material The online version of this article (doi:10.1007/s40750-017-0059-y) contains supplementary material, which is available to authorized users.

Rohan Kapitány

rohankapitany@gmail.com

1 School of Anthropology and Museum Ethnography, University of Oxford, Oxford, UK

2 Early Cognitive Development Centre, School of Psychology,

University of Queensland, Brisbane, Australia 


\section{Introduction}

When Provine (1986) initiated modern academic interest in contagious yawning his interest was in whether the yawn was a human releasing stimulus, a stimulus which, when observed by another, 'releases' or elicits unlearned behaviour (Thorpe 1963; Yoon and Tennie 2010; Zentall 2001). The yawn seemed a likely candidate at a time when few examples had been previously identified in humans (Eibl-Eibesfeldt 1975). Provine established the standard protocol for the observation of yawns: show participants videos (or audio, or written descriptions) of yawns for several minutes and count the number of yawns observed. Then compare this number to the number of yawns observed when participants are exposed to other, equally identifiable stereotypic motor patterns (such as hiccups, sneezes, or laughter). On the face of it, the observation that yawns are contagious was reasonable, with written descriptions dating back at least as far as 300 BCE (Zimara 1580). Provine's interest was what yawning, as a releasing stimulus, revealed about our neurology and our evolutionary history, an interest that has continued to this day (for reviews see: Gallup 2011; Guggisberg et al. 2010, Guggisberg et al. 2011).

In the ethological literature (distinct from the medical literature), a contagion is a shortterm spread of a species-specific behaviour aroused by a specific stimulus (often behaviour) expressed in others in a coordinated manner (Armstrong 1951; Thorpe 1963; Yoon and Tennie 2010; Zentall 2001). Examples may include mating displays, formidability displays, and flocking behavior. A contagious response is a very low-level cognitive response contingent primarily upon one's perception of the stimuli. And yet, perception is a necessary but not sufficient explanation: it is known that yawn contagion is primarily a function of social considerations. We are far more likely to yawn to close family and friends, than we are to strangers or acquaintances (Demuru and Palagi 2012; Norscia and Palagi 2011); indeed, empathy, theory of mind, self-awareness, and psychopathy-related traits are all associated with one's tendency to yawn contagiously (Bartholomew and Cirulli 2014; Norscia et al. 2016; Palagi et al. 2009; Platek et al. 2003; Rundle et al. 2015). Contagious yawning has been observed in non-humans, and is typically associated with that species' social abilities (Demuru and Palagi 2012; Gallup et al. 2015; Madsen et al. 2013; Palagi et al. 2009; Romero et al. 2014). While there are other explanations that are possible alternatives to 'contagion' (Yoon and Tennie 2010), we will not further discuss these alternatives, as we intend to explore the status quo explanation: contagion.

So how seriously should we take the observation that yawns are contagious? Certainly, in groups, humans yawn. Not only do we see a temporal and spatial relation between yawning individuals, but we have an easily identified subjective experience associated with our own personal need to yawn in response to others. And yet in an abstract sense, much like the incorrect observations associated with the hot-hand fallacy (that basket-ball shooters have streaky performance which predicts future behaviour; Burns and Corpus 2004; Gilovich et al. 1985), the observation that yawning is contagious may have arisen as a consequence of our tendency to see patterns and causation where none exists, to misinterpret the clumpiness of randomness as something else. That is, the observation of contagion in groups is a perceptual misunderstanding of randomness, and is a phantom-signal in the noise. While at a personal level, confirmation bias, in which situations that conform to our expectations are salient and memorable while those that fail to are not, could easily (and erroneously) lend weight to the claim that yawns are contagious. Human yawning is associated with the time of day (Zilli et al. 2007), fatigue or boredom (Baenninger and Greco 1991; Provine and 
Hamernik 1986), various medical or psychological afflictions (Daquin et al. 2001; Walusinkski 2009), the temperature of one's brain (Gallup 2011; Gallup and Gallup 2008; Shoup-Knox et al. 2010) and, in experimental contexts, whether or not one is being observed (Baenninger and Greco 1991; Gallup et al. 2016). Indeed, when one's intent is to observe yawn contagion it is famously difficult; Baenninger and Greco (1991) wrote "in our laboratory we have consistently failed to find contagion of yawning when subjects are being openly observed" (pp. 454; that yawns are difficult to observe openly under laboratory conditions - for any researcher - should give pause for concern: for it is the opposite of our everyday experiences and observations - contagious yawns are almost always made or seen when being 'openly observed' in communal places).

Human children yawn, but do not appear to do so contagiously until about the age of 5 or 6 (Anderson and Meno 2010; likewise in chimpanzees, Madsen et al. 2013), and as age increases in adulthood, the likelihood of catching a yawn decreases (Bartholomew and Cirulli 2014). Considering all the apparent boundary conditions associated with the production of both spontaneous and contagious yawning, it may be that the null hypothesis has not been considered seriously enough. Yawns are primarily only measured in the lab, under constrained circumstances after exposing participants to minutes of stimuli. Their co-occurrence in naturalistic settings is contingent upon a range of factors, all of which can produce an apparent sensation of contagion, despite the fact that logic (and statistics) suggest that even if yawns weren't contagious, they may appear as if they were.

Independent of this, there is also a problem with analysis which could lead to an over-estimation of how likely yawns are to occur, and what the typical latency of contagion is. Amidst an increasingly vocal cry for more rigour in statistical and experimental processes in the social sciences (Gelman and Loken 2013; Simonsohn et al. 2014a; Simonsohn et al. 2014b), there are indications that measures don't adequately handle Type-I error, or rule-of-thumb type heuristics are applied to intuitively handle the error. For example, as recently as last year, Gallup et al. (2016) wrote: "Since the rate of spontaneous yawning is quite infrequent, and studies have demonstrated that participants yawn much more frequently when watching a video stimulus depicting yawns compared to control stimuli, we can be confident that the vast majority of yawns reported in the current experiment were contagious [rather than spontaneous]" (pp. 3), and Norscia et al. (2016) that: "...we recorded yawn responses within a 3 min (sic) time window, thus reducing the probability of mistakenly coding spontaneous yawns as yawn responses" (pp. 3; emphasis added). Miscategorization of noise as signal, even within a conservative window, falsely inflates reported rates of focal behaviour. Even in studies where yawns are observed in control conditions, no attempt is made to use this information to inform the accuracy of the observations in test conditions. It is not enough to simply say that the treatment condition produced more yawns than the control, since the control produced yawns at a rate above 0 . How many yawns in the treatment condition, then, were the product of the stimuli, and how many would have been produced independent of the stimuli?. ${ }^{1}$ The rate of Type- 1 error

\footnotetext{
${ }^{1}$ Conversely, one could ask whether the control condition (say, listening to someone breathing ordinarily) also 'releases' yawns, but to a lesser extent? There is an additional line of enquiry rarely examined which involves the contagiousness of the control stimuli: if researchers conduct t-tests on instances of yawning between conditions, ought they not also run the same analysis comparing the number of control condition-specific behaviors elicited between conditions?
} 
should be a serious concern, particularly since methods vary widely. Some studies categorize as contagious any yawn that occurs up to 5-min after a trigger yawn (Madsen et al. 2013; Palagi et al. 2009; Provine 1986), some limit this to 3-min (Demuru and Palagi 2012; Norscia et al. 2016; Norscia and Palagi 2011) and some do not report at all the window in which they consider a yawn spontaneous or contagious (Bartholomew and Cirulli 2014; Platek et al. 2003). Moreover, some authors exclude yawns that occur in the appropriate window of time if they cannot directly attribute it to a single trigger (thus systematically biasing latency measurements and ignoring base-rates; Demuru and Palagi 2012; Norscia et al. 2016; Norscia and Palagi 2011). Independent of this, the difficulty of observing contagious yawns in the wild has led researchers to employ extreme induction methods in the lab. Many studies expose participants to literally minutes of continuous yawning on various media (Bartholomew and Cirulli 2014; Gallup et al. 2016; Madsen et al. 2013; Massen et al. 2015; O'Hara and Reeve 2011; Provine 1986, 1989). Taken together, the measurement and analyses of contagious yawns is far removed from the conditions under which most people believe contagious yawns occur - i.e., when being openly observed among others, after only a brief exposure, and with only a brief latency (see: Results).

One possible solution in the management of these kinds of errors is the use of AgentBased Modelling (ABM). Agent-Based Models simulate interacting agents according to given rules of conduct. In so doing complicated emergent phenomena can be observed and measured. The 'Game of Life', and cellular automata more generally, are excellent and historic examples of this approach (Gardner 1970). An additional benefit of using models to understand social phenomena is that the building of such models requires the model's author to make explicit assumptions that may be hidden using natural language (Marewski and Olsson 2009). For example, spontaneous yawns are produced according to a number of factors, but how frequently do they occur? If I were to observe two 'trigger' yawns in quick succession, am I more likely to yawn contagiously than if I only observed one? What about 3 , or 12 , or 99 ? Does a trigger yawn leave a 'trace' such that I am more likely to contagiously yawn again within a certain time span, and if so, at what rate does the trace degrade? Am I likely to respond to a trigger at a diminishing linear rate, or is the relationship curved? ABM's allow the easy exploration of these questions, as well as management of Type-1 error.

This paper describes one such model (publicly available [upon publication]), which will address various questions: How contagious are yawns? For how long is it appropriate to consider a spontaneous yawn a 'trigger'? And how often do we miscategorise a spontaneous yawn (which necessarily must occur at a base frequency) as a contagious yawn? We also seriously examine the null hypothesis: if yawns are not contagious, might it still look as if they were? The model is first presented along with data it produces based on hypothetical input. Second, we present a behavioural study closely matched to the model's design, in order to ground the input and output variables. Finally, the results are presented by matching real data (from the behavioural experiment) to the agent-based model in order to address (and resolve) empirical and theoretical questions associated with the topic of contagious yawning (with both preregistered and exploratory analyses). 


\section{Methods: the Model}

The model is a cellular automata; a virtual world populated with agents who either yawn, or do not yawn. It is moderately analogous to a group of people sitting quietly in a room waiting for an appointment, passengers on a bus, or students in a classroom. Time proceeds in discrete units (equivalent to 1-min blocks), and the agents are given a base-rate probability of yawning in any given minute (e.g., 1\%). If the random-number generator generates a number below the base-rate, the agent yawns (turns red; see Fig. 1). If an agent is adjacent to a yawning agent within a fixed range (i.e., if an agent can 'see' another agent yawn), the likelihood of yawning increases above base-rate (i.e., there is contagion; e.g., the increased likelihood might now be $2 \%$ ). If the random number resolving yawns is less than the increased base-rate, but greater than the true base-rate (somewhere between $1 \%-2 \%$, for example), the yawn is considered contagious (and is highlighted by a yellow square). If the random number is less than the base-rate, then it can be assumed (probabilistically over the course of time) that the yawn was a spontaneous yawn, but only appears to be contagious by way of temporal and spatial proximity (hereafter referred to as an 'incidental yawn'). For a more comprehensive description of the model see the supplementary material.

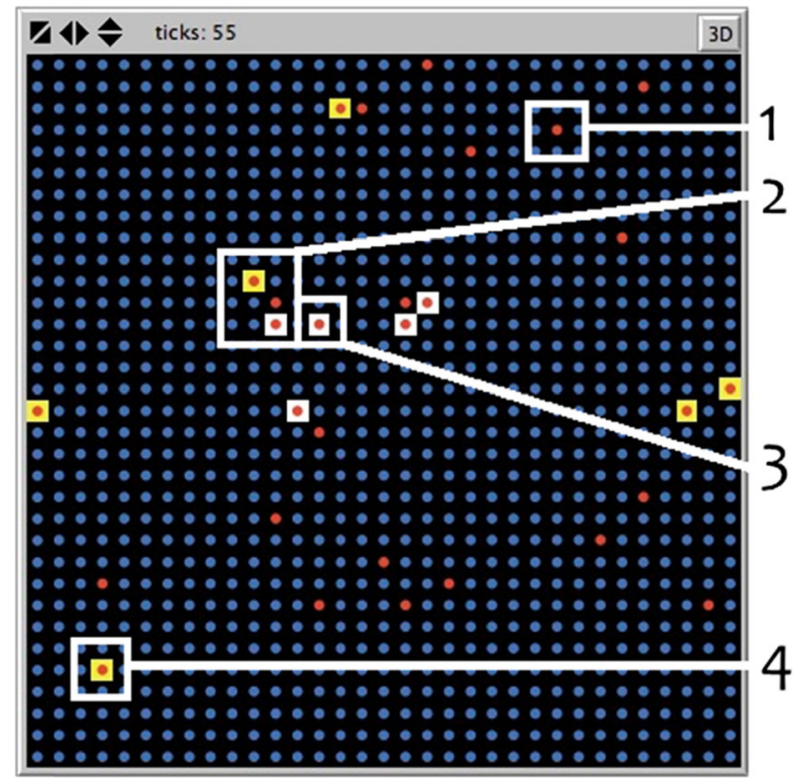

Fig. 1 Example of visual output of the Yawn Contagion Model. Note: Blue agents are agents who are not yawning. Red agents are yawning (1). Agents surrounded by yellow are agents who are contagious yawning as a result of another agent yawning in the very same 'minute' (2) or during a previous 'minute' (according to the latency settings; 4). Agents with a white background are agents who are spontaneously yawning in proximity to a preceding yawn in the very same 'minute' (2) or during a previous 'minute' (3) - these would ordinarily be counted as a contagious yawns, when they are in fact 'incidental'. This world wraps horizontally and vertically, such that agents on the left threshold are functionally adjacent to agents on the right, and the agents on the top are adjacent to agents on the bottom 


\section{Model Data}

In order to demonstrate how the model works, the data it produces, and the potential extent of the operational problems in the yawning literature, we generated some data based on hypothetical input values. We assumed all agents were able to 'see' 8 other agents (this number was chosen arbitrarily, though given that the model acts without error, the number of visible agents is inconsequential. i.e., the model has 'perfect power' to observe the effect). The simulation was run assuming yawns were contagious, and again assuming they were not. We ran our model using 3 yawn-latencies (1-min, 3-min, and 5min) over a total of 120 simulated minutes in order to determine the accuracy of [behavioral] coding methods employed. The simulation was run 25 times for each set of values. Results are described in Tables 1 and 2. Given this is hypothetical data, so for the sake simplicity and interpretation, we have set the base rate of yawns occurring to once-per-hour.

If we accept the extreme null hypothesis (that yawns are not contagious, and the appearance of contagion is an illusion), and assuming a 1-min latency, then $12.10 \%$ of all yawns would be incidental, but would otherwise be incorrectly categorized as contagious. This value explodes to $47.87 \%$ if we assume yawns are contagious for 5 min. Thus, much like the previously mentioned apparent hot-hand effect, the observation that yawns are contagious can easily be made in error - yawns may look contagious, even when they are not.

If yawns are contagious (as the evidence suggests) then the true rate of contagious yawning (at 1 min latency) is $33.20 \%$, with an additional $12.10 \%$ of all yawns identified as incidental (and miscategorised as contagious). With a 5-min latency we are wrong more often than we are right, with $41.87 \%$ of yawns being incidental and only $33.25 \%$ being contagious. Critically, if yawns are actually contagious for $1 \mathrm{~min}$, but we incorrectly assume that yawns are contagious for longer, then these values balloon. If we assume yawns are contagious for longer than they really are, then the true rate of contagion stays constant at around 1/3rd (as per Table 2), but the proportion of incidental yawns increases from a true rate $12.38 \%$ to $30.19 \%$ (for a 3-min latency) and to $41.74 \%$ (for a 5-min latency). Note that these values are very similar to the values in Table 2, but are theoretically different - the data in Table 3 do not allow for [true] contagious yawns to cause other contagious yawns (whereas this relationship is possible in Table 2).

Table 1 Simulated Data in which yawns are not contagious and occur 'once per hour' (chance per tick = .0166) over 120-simulated-minutes (simulated 25 times)

\begin{tabular}{lllllll}
\hline & $\begin{array}{l}\text { Mean } \\
\text { incidental }\end{array}$ & $\begin{array}{l}\text { Mean } \\
\text { contagious }\end{array}$ & $\begin{array}{l}\text { Mean } \\
\text { spontaneous }\end{array}$ & Mean Total & $\begin{array}{l}\text { Percent } \\
\text { incidental }\end{array}$ & $\begin{array}{l}\text { Percent } \\
\text { spontaneous }\end{array}$ \\
\hline Latency 1 & $267.56(16.30)$ & 0 & 1903.32 & $2170.88(47.05)$ & $12.32 \%$ & $87.68 \%$ \\
Latency 3 & $715.80(35.78)$ & 0 & 1470.64 & $2186.44(52.07)$ & $32.74 \%$ & $67.26 \%$ \\
Latency 5 & $1041.16(46.87)$ & 0 & 1133.84 & $1275.00(57.33)$ & $47.87 \%$ & $52.13 \%$ \\
\hline
\end{tabular}

Spontaneous values have been calculated by subtracting incidental and contagious yawns from total yawns. This is why there is no Standard Deviation 
Table 2 Simulation Data in which yawns occur 'once per hour' (chance per tick $=.0166$ ) over 120simulated-minutes (simulated 25 times). Yawns increase the likelihood of subsequent yawns by a factor of 1.5

\begin{tabular}{llllllll}
\hline & $\begin{array}{l}\text { Mean } \\
\text { incidental }\end{array}$ & $\begin{array}{l}\text { Mean } \\
\text { contagious }\end{array}$ & $\begin{array}{l}\text { Mean } \\
\text { spontaneous }\end{array}$ & Mean total & $\begin{array}{l}\text { Percent } \\
\text { incidental }\end{array}$ & $\begin{array}{l}\text { Percent } \\
\text { spontaneous }\end{array}$ & $\begin{array}{l}\text { Percent } \\
\text { contagious }\end{array}$ \\
\hline Latency 1 & $\begin{array}{l}392.24 \\
(22.55)\end{array}$ & $\begin{array}{l}1076.84 \\
(27.64)\end{array}$ & 1774.24 & $\begin{array}{l}3243.32 \\
(50.10)\end{array}$ & $12.10 \%$ & $54.70 \%$ & $33.20 \%$ \\
Latency 3 & $\begin{array}{l}982.4 \\
(36.53)\end{array}$ & $\begin{array}{l}1084.96 \\
(32.84)\end{array}$ & 1197.88 & $\begin{array}{l}3265.24 \\
(42.43)\end{array}$ & $30.09 \%$ & $36.69 \%$ & $33.23 \%$ \\
Latency 5 & $\begin{array}{l}1360.12 \\
(42.23)\end{array}$ & $\begin{array}{l}1082.40 \\
(31.07)\end{array}$ & 813.08 & $\begin{array}{l}3255.60 \\
(55.19)\end{array}$ & $41.78 \%$ & $24.97 \%$ & $33.25 \%$ \\
\hline
\end{tabular}

Spontaneous values have been calculated by subtracting incidental and contagious yawns from total yawns. This is why there is no Standard Deviation

\section{Discussion}

While it is the case that the values derived were based on the hypothetical value of 1-yawnper-hour, it would not matter whether we used 1-, 10-, or 100-yawns-per-hour, as the pattern of output would be functionally equivalent. To the best of our knowledge, no such estimate exists regarding how contagious a yawn is (i.e., to what extent a trigger influences yawn production above base-rates in those who observe it), and has been chosen conservatively. These data demonstrate two things: First, even if yawns are contagious, Type-1 error is still a serious problem. Second, the null hypothesis needs to be taken seriously - it remains entirely possible that the phenomenon of contagious yawning, as observed since antiquity, is illusory. While this may not be the case, the fact this has not been acknowledged suggests a failure in our empirical practices. The following behavioral experiment replicates, as best as possible, an ecologically valid environment in which contagious group yawns occur, in order to produce data to feed into the model.

\section{Methods: the Behavioral Experiment}

\section{Participants}

Undergraduate students $(N=79)$ at a large Australian University made up the final data set, and participated in a total of 16 sessions (on average, 4.94 students per group

Table 3 Hypothetical Data in which yawns occur 'once per hour' (Base-Rate = 1.666) over 120-simulatedminutes (simulated 25 times). Yawns increase the likelihood of subsequent yawns by a factor of 1.5 only in for a 1-min latency. Data assumes a latency of 3- and 5-min

\begin{tabular}{|c|c|c|c|c|c|c|c|}
\hline & $\begin{array}{l}\text { Mean } \\
\text { incidental }\end{array}$ & $\begin{array}{l}\text { Mean } \\
\text { contagious }\end{array}$ & $\begin{array}{l}\text { Mean } \\
\text { spontaneous }\end{array}$ & $\begin{array}{l}\text { Mean } \\
\text { total }\end{array}$ & $\begin{array}{l}\text { Percent } \\
\text { incidental }\end{array}$ & $\begin{array}{l}\text { Percent } \\
\text { spontaneous }\end{array}$ & $\begin{array}{l}\text { Percent } \\
\text { contagious }\end{array}$ \\
\hline Incident Latency 3 & $\begin{array}{l}988.68 \\
(29.77)\end{array}$ & $\begin{array}{l}1092.12 \\
(34.32)\end{array}$ & 1193.801 & $\begin{array}{l}3274.6 \\
(63.03)\end{array}$ & $30.19 \%$ & $36.46 \%$ & $33.35 \%$ \\
\hline Incident Latency 5 & $\begin{array}{l}1362.88 \\
(47.53)\end{array}$ & $\begin{array}{l}1088.64 \\
(35.63)\end{array}$ & 813.081 & $\begin{array}{l}3264.60 \\
(47.00)\end{array}$ & $41.74 \%$ & $24.91 \%$ & $33.35 \%$ \\
\hline
\end{tabular}


session). The sample contained 61 women (77.2\%) with a reported age range between 17 and 29 years $($ mean $=19.65, \mathrm{SD}=2.38)$. Participants received credit in exchange for their time. Data from two additional sessions, which included a total of 10 participants, were excluded from analyses due to disruptive behaviour from one participant in each session, respectively. Of the 16 sessions that were analysed, 10 sessions were conducted in the morning, and 6 were conducted in the afternoon. All participants were verbally briefed, and written consent was obtained prior to participation. Participants indicted in writing whether their video data could be made public.

\section{Experimental Design and Protocol}

This was a within-subjects design with two levels of one factor. Participants sat listening to an audio programme on their personal devices while wearing a blind-fold, or not wearing a blind-fold. Participants arrived at a pre-specified time, and were tested as a group. A total of 16 groups were tested. On average, each group had 4.94 members (with a mode and max of 6, and a single minimum of 3 ). Upon arrival participants were asked to sit in chairs arranged around a table facing inwards (thus, each participant could potentially see all others when not wearing a blind-fold). They were told they would be asked to remain seated for the duration of the experiment. Then they were asked to listen to Chopin's Complete Nocturnes using earphones on their personal electronic devices (iPhones, Androids, etc.). This was done for two reasons: first, in the event that someone yawned during the blind-fold condition the music/earphones prevented them from hearing it (which has been shown to elicit a contagious yawn; Massen et al. 2015), and second, participants interest levels were kept relatively constant. The order of blind and non-blind conditions was counterbalanced over testing sessions ( 7 groups were blind-folded in the first session, 9 groups were blind-folded in the second). After $25 \mathrm{~min}$ of the first session participants were asked to either remove or don their blindfolds, depending on condition. At the completion of the testing session participants filled out a brief survey assessing to what extent they found the music interesting, how sleepy they were, their recollection of whether they yawned in the preceding hour, and self-report measures of their own tendency to yawn contagiously (see Supplementary Material for full list of measures)

While evidence shows that being observed moderates the rate of yawning, participants were informed (in the written brief sheet) that they were being filmed. Two cameras were placed inconspicuously in the testing room. The experimenter sat at a remove from the group, facing a wall. At no point prior to the survey was any cue given that the experiment was about yawning (i.e., the experimenter did not yawn, and the recruiting and briefing process gave no indication as to the research topic).

\section{Behavioral Coding}

The lead author coded the behaviour of all participants based on the video data (available in full online at [available upon publication or request]. Each time a participant yawned it was counted and time-stamped. A second blind researcher coded the dataset in it's entirety. Reliability was extremely high in both session (First Session alpha $=.980$, Second session alpha $=.959)$. 


\section{Pre-Registered Analyses and Results}

The following pre-registered analyses and predictions were made: Analyses 1 (A1) and 2 (A2), respectively, are t-tests between 'interest' in both session 1 and 2, as well as on 'boredom'. Analysis 3 (A3) is a t-test between non-blind yawns in session 1 and 2, and Analysis 4 (A4) is a t-test between blind yawns in session 1 and 2. Analysis 5 (A5) was a t-test on all yawns in session 1 and session 2 (ignoring condition information). Analysis 6 (A6) was a t-test of yawns-per-minute between blind and non-blind conditions. Descriptive statistics are presented based on self-report measures.

All the following questions were answered at the completion of both sessions. On two separate 10-point scales (where 1 was 'Not bored/interested', 10 was 'Very bored/ interested', and 5 being 'moderately bored/interested'), paired-samples t-tests revealed that participants thought the first session was more interesting $(M=4.83$, SD 2.24) than the second $(\mathrm{M}=4.22, \mathrm{SD}=2.33), \mathrm{t}(77)=2.242, p=.028$ (A1). Reflecting this, the second session was more boring $(\mathrm{M}=6.35, \mathrm{SD}=2.51)$ than the first $(\mathrm{M}=5.28$, $\mathrm{SD}=2.33), \mathrm{t}(77)=3.332, p=.001(\mathrm{~A} 2)$.

To determine whether counter-balancing worked, we aggregated all yawns from all individuals into bins of one-minute. There was no significant difference between the average number of yawns produced per minute in Session $1(\mathrm{M}=.033, \mathrm{SD}=.10)$ and Session $2(\mathrm{M}=.048, \mathrm{SD}=.09), \mathrm{t}(78)=.969, p=.336(\mathrm{~A} 5)$. We found there was no difference in the mean number of yawns-per-minute when participants were blindfolded in Session $1(\mathrm{M}=.15, \mathrm{SD}=.54)$ compared to Session $2(\mathrm{M}=.40, \mathrm{SD}=.84)$, $\mathrm{t}(66.70)=1.552, p=.125(\mathrm{~A} 4)$. There was, however, a difference in the mean number of yawns per minute when participants were able to see one another in Session 1 $(\mathrm{M}=.90, \mathrm{SD}=1.58)$, compared to Session $2(\mathrm{M}=2.03, \mathrm{SD}=2.95), \mathrm{t}(57.85)=2.105$, $p=.04$ (A3), such that participants who saw each other in Session 2 (rather than Session 1) yawned more frequently (discussed further in 'Non-registered analyses and results').

Overall, we found that when participants were blind-folded they produced a mean of .011 yawns-per-minute ( $\mathrm{SD}=.03$ ), or .667 yawns-per-hour; when not blind-folded, participants produced a mean of .058 yawns-per-minute $(\mathrm{SD}=.10)$, or 3.48 yawns-perhour, and this difference was significant, $\mathrm{t}(78)=4.60, p<.001$ (A6). Figure 2 shows the total number of yawns per minute for each condition.

We also tested assumptions of the literature against beliefs of the participants. Seven of 79 people $(8.9 \%)$ reported that yawns were not contagious; all others reported they were contagious. When asked "How likely are you to yawn if you see someone [else] yawn?", participants responded that they were, on average, $100.13 \%$ more likely to yawn (i.e., twice as likely; SD =342.15). On average, participants believed that they would yawn in response to a trigger yawn typically after $29.84 \mathrm{~s}(\mathrm{SD}=34.66 \mathrm{~s})$, and that the cut-off for someone being able to 'catch' a yawn was, on average, $112.16 \mathrm{~s}$ (less than $2 \mathrm{~min}$; SD = $171.02 \mathrm{~s})$. When blindfolded, a total of 67 of $79(84.8 \%)$ did not yawn at all, and when not wearing a blind-fold, a total of 46 of $79(58.2 \%)$ of participants did not yawn at all. Figure 2 shows the frequency of yawns. Of the 38 people who yawned at least once during the experiment, only $8(21.05 \%)$ claimed that during the experiment at least one of their yawns was 'caught' from someone else. When asked to remember how many yawns they produced during the experiment, the difference between their estimate $(\mathrm{M}=2.17, \mathrm{SD}=2.72)$ was not significantly different 


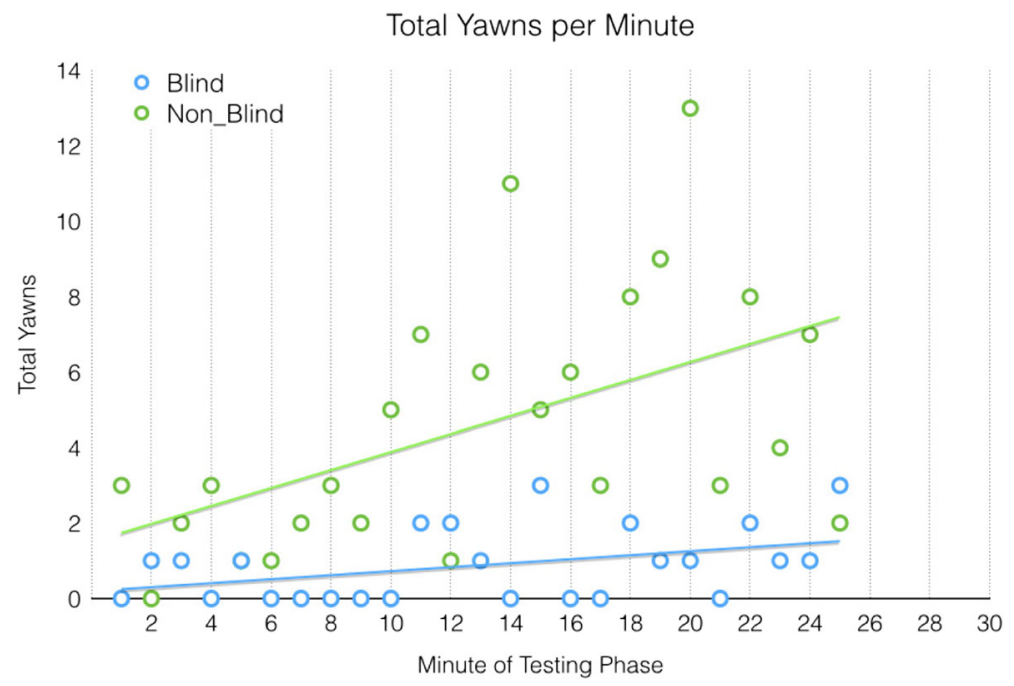

Fig. 2 Total Yawns occurring per each minute

from an objective count $(\mathrm{M}=1.96, \mathrm{SD}=3.45), \mathrm{t}(77)=.574, p=.567$. There was no systematic pattern of correlations between any of these values (as well as boredom and interest) with total number yawns produced overall, or in specific sessions.

\section{Non-registered Analyses and Results}

Determining that people yawn more frequently in the non-blind condition than the blind condition does not necessarily indicate contagion. It may simply mean the environment is conducive to frequent yawning. We conducted an autocorrelation to determine whether the presence of a yawn at Time- $\mathrm{N}$ is correlated at Time- $(\mathrm{N}+\mathrm{x})$, where $\mathrm{x}$ is increasing units of time. In the blind condition, we found that yawning at $\mathrm{T} 0$ did not correlate with/predict yawns at any other time point (Fig. 3a). We found a number of auto-correlations in the non-blind condition, but not at intuitive time-lags, although the overall trend is self-evident (Fig. 3b).

As suggested by prior analyses, we tested for an interaction to confirm the finding that the likelihood of contagiously yawning increases when participants were able to see each other in the second phase of the experiment. Corroborating our pre-registered analyses, we found no main effect of order in which participants wore a blind-fold on yawns-per-minute, $\mathrm{F}(1)=2.517, p=.115$. As with our prior analyses, we found a main effect of wearing a blind-fold, $\mathrm{F}(1)=18.306, p<.001$. As suggested by our manipulation checks, we found a significant interaction, $\mathrm{F}(1)=6.124, p=.014$. Looking at mean yawns-per-minute, this interaction reveals that participants who could see each other in the second session $(\mathrm{M}=.081, \mathrm{SD}=.118)$ yawned more than those who could see each other in the first session $(\mathrm{M}=.036, \mathrm{SD}=.063$; See Fig. 4).

To produce comparable data to other experiments, we used the standard protocol of identifying trigger yawns and counting all subsequent yawns. Specifically, we followed and modified the protocol of Demuru and Palagi (2012) as they outline the protocol 
a

0.4

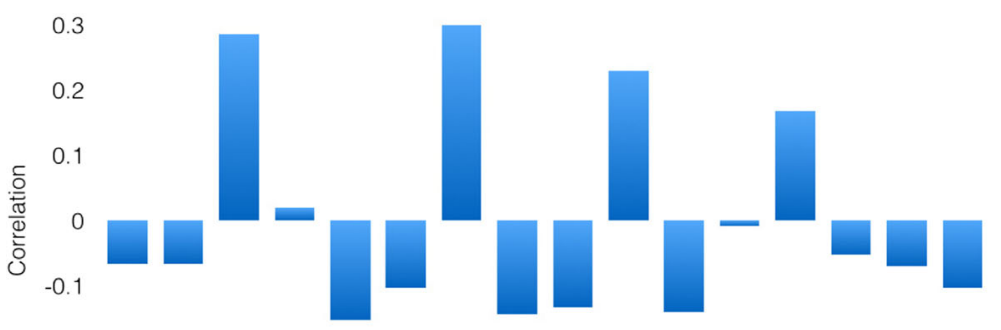

$-0.2$

$-0.3$

$-0.4$

\begin{tabular}{llllllllllllllll}
\hline 1 & 2 & 3 & 4 & 5 & 6 & 7 & 8 & 9 & 10 & 11 & 12 & 13 & 14 & 15 & 16 \\
Time Lag & & & &
\end{tabular}

Autocorrelations of Yawning across Time-lags in the non-Blind

b

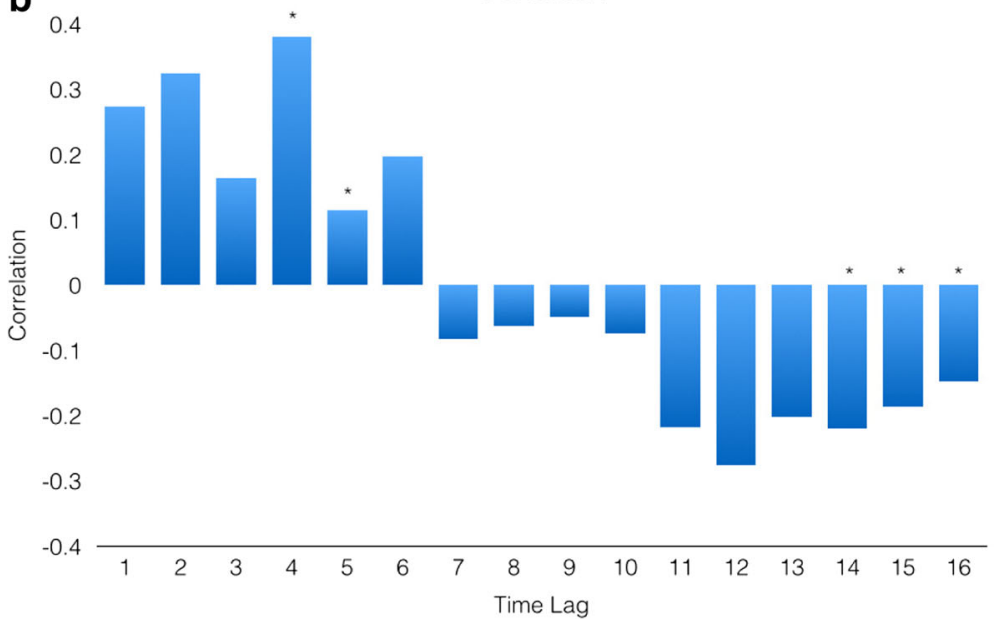

Fig. 3 a and $\mathbf{b}$ Auto-correlations of yawns at increasing time-lags. $*<.05$

most clearly, and have a similar dataset. The criteria were modified because, while the original criteria were applicable in the blind-condition (in which the yawn frequency was low), they resulted in too many omissions in the non-blind condition to provide representative results (original criteria in italics). The criteria are as follows:

- A trigger yawn is any yawn that occurs, and which precedes another yawn from another person, within the specified latency, and which itself was not triggered; $A$ contagious yawn is any yawn that occurred subsequent to a trigger yawn within the specified latency. Contagious yawns are only counted once, even if they could have multiple triggers; If a contagious yawn resulted in another person yawning subsequently, it is also considered a trigger yawn; A Spontaneous yawn is a yawn that is 


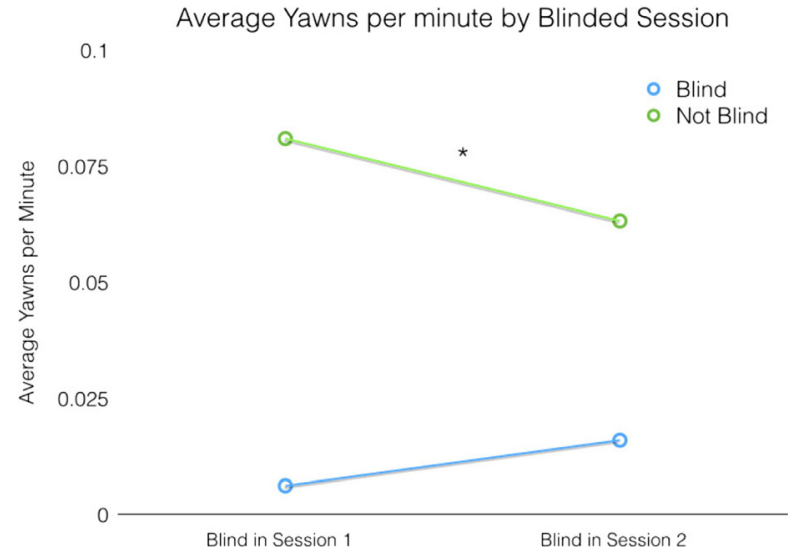

Fig. 4 An interaction between wearing a blind fold, and order in which a blind fold is worn

neither a trigger yawn, nor a contagious yawn. An individual who, for example, yawns 4 times in $4 \mathrm{~min}$, but does not arouse another to yawn, has produced 4 spontaneous yawns; If two people yawned at time $\mathrm{x}$, and another person yawned at time $(\mathrm{x}+$ latency), then the first two yawns were both considered triggers.

We found that within the non-blind condition the number of 'contagious yawns' more than doubles if the latency is increased from 1-min to 5-min (from $28.7 \%$ to $60.0 \%$ ). The same method of attributing contagion in the blind condition suggests that $4.45 \%$ of yawns are contagious at 1-min (when actually they can only be incidental), and more-than-doubles to $13.64 \%$ at 5-min - a number which simply cannot reflect reality. See Table 4 for all values.

An alternative question to ask is 'how often do untriggered yawns trigger someone else to yawn?'. That is, what proportion of yawns that are not caused by other yawns (i.e., all spontaneous and all trigger yawns, as per Table 4) appeared to cause another to yawn within the specified latency? Table 5 shows this value, where the absolute number of trigger yawns has been modified by half the value of the overlap column in order to prevent falsely inflating the result. Here, we find a similar, but more extreme,

Table 4 Categorization of yawns based on experimental data

\begin{tabular}{llllll}
\hline & Latency & $\begin{array}{l}\text { Spontaneous } \\
\text { Yawns }\end{array}$ & $\begin{array}{l}\text { Trigger } \\
\text { Yawns }\end{array}$ & $\begin{array}{l}\text { Contagious } \\
\text { Yawns }\end{array}$ & $\begin{array}{l}\text { Trig / Cont } \\
\text { Overlap }\end{array}$ \\
\hline Non-Blind & 1-Minute & $56(48.70 \%)$ & $36(31.30 \%)$ & $33(28.70 \%)$ & 10 \\
$N=115$ yawns & 3-Minute & $29(25.22 \%)$ & $58(50.43 \%)$ & $59(51.31 \%)$ & 31 \\
Blind & 5-Minute & $19(16.52 \%)$ & $73(63.48 \%)$ & $69(60.00 \%)$ & 46 \\
$N=22$ & 1-Minute & $20(90.91 \%)$ & $1(4.55 \%)$ & $1(4.55 \%)$ & 0 \\
yawns & 3-Minute & $17(77.27 \%)$ & $4(18.18 \%)$ & $2(9.09 \%)$ & 1 \\
\hline
\end{tabular}

Totals of Spontaneous, Trigger, contagious yawns do not necessarily sum to $100 \%$ as there is overlap between trigger and contagious yawns. The absolute number of yawns that were double counted is listed in the 'overlap' column 
Table 5 The percentage of yawns that 'cause' subsequent yawns

\begin{tabular}{lllll}
\hline & Latency & Spontaneous Yawns & Trigger Yawns* & $\begin{array}{l}\text { Proportion of } \\
\text { causal yawns }\end{array}$ \\
\hline Non-Blind $N=115$ yawns & 1-Minute & $56(48.70 \%)$ & $31(26.96 \%)$ & $35.63 \%$ \\
& 3-Minute & $29(25.22 \%)$ & $42.5(36.96 \%)$ & $52.15 \%$ \\
Blind $N=22$ yawns & 5-Minute & $19(16.52 \%)$ & $50(43.48 \%)$ & $72.46 \%$ \\
& 1-Minute & $20(90.91 \%)$ & $1(4.55 \%)$ & $4.76 \%$ \\
& 3-Minute & $17(77.27 \%)$ & $3.5(15.91 \%)$ & $17.07 \%$ \\
5-Minute & $16(72.72 \%)$ & $4(18.18 \%)$ & $20.00 \%$ \\
\hline
\end{tabular}

*Trigger yawns have been down-modified by half the value of the overlap column from Table 4

pattern of results. This method of counting demonstrates the discrepancy between erroneously assuming causation (as revealed by the rates in the blind condition), and the rates arrived at when causation is in principle possible.

Similar to the data presented in Tables 4 and 5 shows that within the non-blind condition the number of 'trigger yawns' more than doubles if the latency is increased from 1-min to 5-min (from $35.63 \% \%$ to $72.46 \%$ ). The same method of attributing triggers in the blind condition suggests that $4.76 \%$ of yawns are triggered within 1-min (when actually they can only be incidental), and more-than-quadruples to $20.00 \%$ at 5$\min -$ again, this is a number which simply cannot reflect reality.

\section{Analyses as a Result of Peer-Review}

As familiarity and time of testing can impact contagious yawning, we also evaluated whether the extent participants knew each other and session time was associated with yawning patterns.

Across a total of 79 participants, only 13 reported they had friends in the same testing session. Of the 16 sessions, 10 were conducted in the morning (beginning at either 1000 or $1030 \mathrm{~h}$ ) and 6 in the afternoon (beginning at 1500 or $1600 \mathrm{~h}$ ). In order to determine the influence of these factors on the number of yawns produced by individuals, a MANOVA was performed, in which total number of yawns produced when Blind and Non-Blind was (were) the dependent variable(s), and the binary values of 'friends present', and 'time of day' were entered as a random variable. When Blind, the influence of friends was non-significant $\mathrm{F}(1)=.380, p=.539$, as was the influence of time of day, $\mathrm{F}(1)=.000, p=.985$. When Non-Blind, the influence of friends was nonsignificant, $\mathrm{F}(1)=.000, p=.992$, as was the influence of time of day, $\mathrm{F}(1)=.224$, $p=.637$. We thus find no evidence that the presence of friends, or the time of day, influenced the total number of yawns individuals produced in either condition.

We conducted a reliability analysis within yawners between blind and non-blind conditions, which produced an alpha $=.308$. Descriptively, of 12 people who yawned at least once in the blind condition, all but 3 yawned again in the non-blind condition. Of the 67 participants who produced no yawns in the blind condition, a total of 24 produced yawns in the non-blind condition. A total of 43 (of 79) participants produced 
no yawns in either condition. Using an a priori method of power analyses, with $\mathrm{n} 1=39$ and $\mathrm{n} 2=40$, we had $80 \%$ power to detect an effect of size $\mathrm{d}=.64$.

\section{Discussion}

Data from the blind-folded session revealed that participants produced, on average, two-thirds a yawn per hour, a rate comparable to the rates reported by Baenninger and Greco (1991) (in their control conditions in experiment 1) and Zilli et al. (2007). When participants could see each other, they produced considerably more yawns (about 3.5 yawns per hour, or one yawn every 15-20 min), particularly when they could see each other in the second session rather than the first. This can most easily be seen in Fig. 4. We found no pattern of auto-correlations when participants were blind-folded, indicating that a trigger yawn at $\mathrm{T} 0$ does not predict subsequent yawns after any amount of time (as expected, given that 'contagion' was not possible). When participants could see each other, we found an odd pattern of auto-correlations. The literature to date suggests that yawns are contagious for up to 5-min, and that yawns are more contagious at low latencies than high latencies (Norscia and Palagi 2011). The auto-correlations here suggest that a trigger at T0 positively predicts yawns at 4-min $(r=.38)$ and 5 -min $(r=.12)$. It seems somewhat absurd that yawns are only contagious after $4 \mathrm{~min}$, and not at all contagious in the first $3 \mathrm{~min}$; this is neither supported by the literature, nor do the majority of participants believe it (whom, we assume, are representative). In investigating this pattern of results, we observed that over $95 \%$ of all yawns produced by individuals occurred within a 5-min window of another of their own yawns - that is, participants who yawned tended to yawn in clusters within a 5-min window, and nearly all yawns observed conformed to this pattern. It seems likely that the pattern of autocorrelations indicates that after yawning once, people are likely to yawn again 4 to 5 min later. If this interpretation is correct, then the fact that the autocorrelation identified it, but failed to identify an autocorrelation at shorter latencies, suggests that yawns are simply not contagious in the way they are commonly believed to be.

Given that yawns increase over-time, more so when we can see other people, and that the presence of a yawn at any given moment does not reliably or intuitively produce a yawn at subsequent brief latencies, we are forced to conclude that the influence of another person's single yawn on one's own likelihood to yawn is, at best, trivial. It is clear from this data that shared environmental factors and the saliency of the social situation, as well as individual differences in yawn-production, are vastly more accountable for the apparent phenomenon of yawn contagion. It also explains the apparent requirement of the literature to saturate participants with minutes of yawning-stimulus in order to elicit the effect, and to maintain a very liberal window in which yawns are considered contagious.

While there is empirical evidence that familiarity and time of day influence the frequency of yawn production, our behavioral experiment did not find this. It is important to note in this context that our experiment was not designed to examine these relationships (indeed, we recruited as randomly as we could) and the difference in time between morning and afternoon session (between approximately $1000 \mathrm{~h}$, and $1500 \mathrm{~h}$ ) was not particularly wide. A richer interpretation may nevertheless hold: if yawns are not contagious, then retreating into a myriad moderators and mediators is 
akin to fishing for significance. Alternatively, if it is the case that time of day and the quality of social relationships impact on the production of contagious yawns (which we do not challenge here), then our failure to observe it may suggest the effect is not as large as implied by the prevailing literature, and additional work needs to be conducted in order to quantify it. We suggest that the use of additional and increasingly sophisticated methodological tools could address this; standard between-participants test/ control designs may not be sufficient to capture the true nature of the relationship. Here, we hope to have demonstrated that various assumptions of the purported effect need to be addressed before demarcating how the effect varies under specific conditions.

One criticism of the behavioral manipulation is that there was no control over how often, and how totally, each participant could observe all other participants. While it may be possible to retrospectively examine this, we suggest it is not necessary (though the videos are made publicly available on the Open Science Framework should others wish to pursue it). The room(s) in which participants completed the task were - as much as possible - devoid of other stimuli. The blinds on the windows were closed, the tables were cleared, phones and laptops were face down or turned off, and all paper was turned face down. We attempted to keep interest and boredom approximately equal for all participants (by way of the audio stimuli). It is possible that some participants spent some of the time looking at the floor, or avoided eye contact. But we point to the clear difference in yawn production to alleviate this concern - people yawned more frequently when they were able to see each other than when they could not. If those who support the claim that yawns are contagious accept this observation as true, but doubt the values obtained because participants weren't required to stare at each other, then the burden of evidence is on them to explain this alternative mechanism of social yawn production: not only are yawns contagious, but yawns are also produced more frequently in a non-contagious manner in the presence of humans they can see, but choose not to. Let us once again appeal to the contexts in which we find ourselves apparently susceptible to contagious yawns: When sitting on a bus, in a waiting room, in a lecture theatre, or sitting in a café, we are able to see some finite number of others in our environment, a value that varies of time, and should we spy another yawning, we may ourselves produce a yawn in response. Certainly we may miss some yawns produced by others, but not all. In this way we believe the current experiment is, for a controlled experimental situation, sufficiently ecologically valid.

Regarding the observation that participants yawn more frequently when they can see each other after being blind-folded than before, a lean interpretation (favoured by the authors) is that yawns tend to increase over time (at least over the duration of the present experiment). We accept that people yawn more often when they can see each other, and so we simply suggest (a hypothetical) interaction between time (possibly a consequence of boredom, which also increased between session 1 and 2) and awareness of others. An alternative is that the state-change associated with the introduction of light, produced increased cortical arousal, which in turn, produced a greater number of spontaneous yawns (Seki et al. 2003). However, given that there was a main effect associated with being un-blinded, one that was not easily described by an autocorrelation, we chose not pursue this difference further (since the implication on contagious yawning are unclear - does either explanation lead to greater susceptibility to contagion?). This may also explain the observation of a greater number of 
spontaneous yawns with a 1-min latency were observed in the non-blind condition relative to the blind condition (despite counter-balancing the order of these conditions). The reasons such an observation is interesting is that, with a low latency, there ought to be fewer yawns attributable to contagion, thus, a high value here might indicate contagion exists and spontaneous yawns are being incorrectly defined as such. There are three possible explanations for this observation. First - and as described above - we simply produce more yawns in social contexts (i.e., when we can see others) than in non-social contexts. Second, this difference is due to the fact that yawns are contagious, but at latencies greater than accounted for in the data (i.e., at latencies greater than five minutes). We find this second explanation unlikely, since the autocorrelation reveals a negative relationship after six-minutes, and this explanation does not shed any light on the observation regarding the greater number of yawns when the non-blind condition came after the first. Third, yawns are contagious, but for whatever reason the autocorrelation failed to detect this relationship. We do not think this third explanation is particularly parsimonious, but a more highly-powered replication with the given methodology might provide support for this position. However, we do not think this is the case, and generally favour the first explanation: That is, we produce more yawns in social contexts, even though yawns themselves may not cause additional yawns in others.

A final criticism (raised in the peer review process) is that the rate of 3.5 yawns per hour observed in the unblinded condition is greater than is typically observed and reported, and as such, calls into question the ecological validity of our study. The number of yawns per hour found here may be unprecedented by virtue of the fact that no published experimental study (to the best of our knowledge) has replicated the conditions of the 'waiting room' (or the bus, or other similar environments). If our participants were left to freely interact with each other, or distract themselves with their phones (for example), our observed rates almost certainly would have been lower and hence more similar to rates reported in non-experimental observational studies where the actions of participants are left to vary in unconstrained and unreported ways. Instead, we argue that our results are ecologically valid and apply to a set of environmental conditions experienced frequently and, rather than being dismissed as unrepresentative, should be regarded as providing additional perspective on this complicated issue.

\section{Fitting the Data to the Model}

The mean number of participants per group in the behavioural experiment was 4.94, who, when they could see each other, yawned at a rate of .0582 yawns-per-minute (or 3.492 yawns-per-hour). Since it is not common for individuals to consider themselves in the number of people they observe, the model was set so that each agent could see 4 others in their immediate neighbourhood. Because the base-rate of yawns (in the blind condition) was .011 yawns-per-minute, the base-rate of yawns was set such that agents have a $1.111 \%$ chance of yawning on any given tick. There were 1089 agents in the model, each with a $1.111 \%$ of yawning in any given minute, over a period of 2-h: thus, we would expect to observe 1452.72 yawns in total. We observed, over 75 independent runs, a mean of $1459.56(\mathrm{SD}=38.50)$. 
As can be seen in Table 6, the simulation data of the blind condition matches very well at 1-min latency. The higher values at greater latencies in simulation data simply reflect the increased power of the model; the behavioural experiment generated far less data, and so likely is an underestimate of the population mean. Additionally, in our 25 simulations all agents could always see 4-other agents (i.e., agents were in groups of 5), in the behavioural experiment, this value varied between 3 and 6 .

Despite the fact that our analyses of behavioural data suggest that yawns are not contagious, there was a difference between groups. One experiment alone cannot undermine the claim that yawns are contagious, and so we will optimize the model on the assumption that the increase in yawns is due to contagion, and not due to other factors (such as those previously described). The process of optimization is simple: At a base-rate of .667 yawns-per-hour, we expected (and observed) that 1089 agents should produce 1452.73 yawns over the 2-h period. When participants in the behavioural experiment could see each other, they produced 3.492 yawns-per-hour, and so we ought to expect to see 7605.576 yawns. Thus, we simply searched through parameters to find a contagion value that produced this output without altering any other values. We found, over 25 independent runs, a contagion factor of 2.665 produced a mean number of 7608.64 $(\mathrm{SD}=82.33)$ yawns when yawns were contagious for 1-min; we found a contagion factor of 2.657 produced a mean number of $7605.80(\mathrm{SD}=79.77)$ when yawns were contagious for 3-min, and a contagion factor of 2.649 produced a mean number $7599.88(\mathrm{SD}=84.85)$ when yawns were contagious for 5-min. Table 7 shows the proportion of contagious and incidental yawns according under these conditions. We observed that, when yawns are considered contagious at a latency of $1-\mathrm{min}, 7.96 \%$ of those considered contagious are actually incidental, at 3-min this value is $19.14 \%$, and at 5-min, the proportion of incidental yawns miscategorised as contagious is $26.03 \%$.

\section{General Discussion}

Do humans in groups yawn in each other's presence more often than when alone? Certainly. Our subjective and affective observations suggest so, and both this data and a history of (somewhat contrived) historic experiments have demonstrated that the observation of yawns leads to an increase in yawning. Is yawning contagious (does the observation of a trigger yawn cause one to yawn in response, in an unlearned

Table 6 Mean values of 25 simulations of the Blind condition where the base-rate $=.0111$ per tick, where agents can see 4 other agents, with no contagion, over 120 -simulated-minutes

\begin{tabular}{lllll}
\hline & Latency & $\begin{array}{l}\text { Spontaneous } \\
\text { Yawns }\end{array}$ & $\begin{array}{l}\text { Incidental Yawns } \\
(\mathrm{SD})\end{array}$ & Total Yawns (SD) \\
\hline Simulation Data & 1-Minute & $1344(95.39 \%)$ & $64.92(7.68)(4.61 \%)$ & $1408.92(38.94)$ \\
& 3-Minute & $1278.68(87.25 \%)$ & $179.68(17.79)(12.75 \%)$ & $1458.36(41.55)$ \\
& 5-Minute & $1219.48(79.28 \%)$ & $291.92(80.69)(20.72 \%)$ & $1511.4(35.00)$ \\
Behavioral Data (Blind & 1-Minute & $20(90.91 \%)$ & $1(4.54 \%)$ & 22 \\
Condition) & 3-Minute & $17(77.27 \%)$ & $2(9.09 \%)$ & \\
& 5-Minute & $16(72.72 \%)$ & $3(13.64 \%)$ & \\
\hline
\end{tabular}




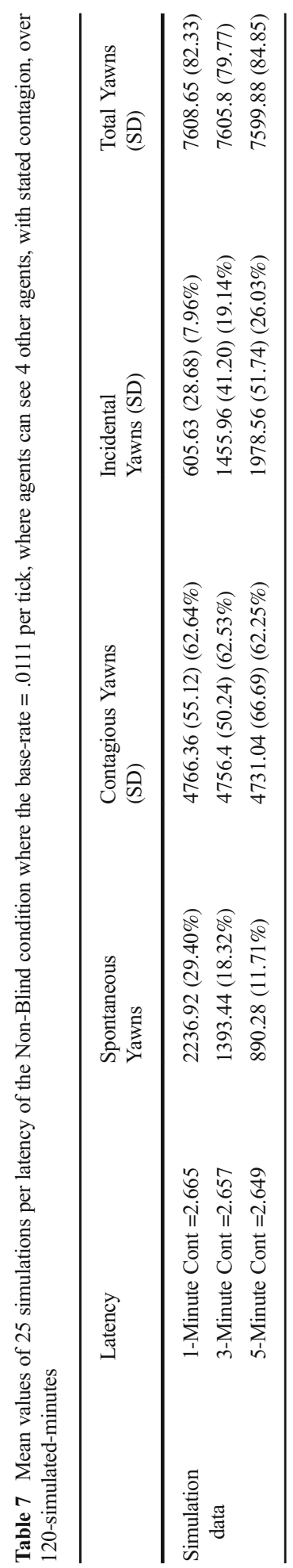


manner)? Maybe. An auto-correlation (which takes input at one time and correlates it with input with a time-lag) suggests that yawns are not immediately contagious, and simultaneously indicates we tend to yawn in clusters (indeed, 95\% of all recorded yawns in the non-blind condition occurred within 5-min of a yawn made by the very same person). Perhaps more critically though, it is demonstrated here that, even if yawns were not contagious, it would be an easy and predictable mistake to make to conclude they were (an important observation, one that has no precedent, and ought to stand as evidence that the field has a blind-spot). If yawns are contagious (and let's assume they are) then it is also the case that we have a long and documented history of willfully failing to account for the miscategorization of contagious yawns (by failing to assume that yawns are produced at a base-rate). This has two implications. First, we have over-estimated the magnitude and meaning of a contagious yawn. Not only do we often risk calling a spontaneous yawn contagious when it is actually incidental, we also omit from analyses a variety of data which is too difficult to appropriately categorize. This has led to the second problem; By using low quality data in our theorizing on the evolutionary significance of contagious yawns (primarily in Homo), we may have been doing little more than seeing faces in clouds or reading tea-leaves.

To the extent that assumptions of the model presented here seem insufficient or unreasonable, we respond that we merely formalized (in a programming language) assumptions extant in the literature. We acknowledge that yawns appear to vary throughout the day and according to one's chronotype (Zilli et al. 2007). Further, we acknowledge that individual differences associated with social awareness or mentalizing also bear upon one's tendency to contagiously yawn (Bartholomew and Cirulli 2014; Demuru and Palagi 2012; Norscia et al. 2016; Norscia and Palagi 2011; Palagi et al. 2009; Platek et al. 2003; Rundle et al. 2015). We acknowledge that some existing experimental protocols are used to mitigate the influence of social factors. And yet, we suggest that the appropriate response to our provocative position is not to appeal to our omission of these factors and dismiss our claims, but rather, to accept that this simple model can be improved to better account for these important variables in the future. This model and experiment should be the starting point in re-examining and attempting to falsify this phenomenon; it is not intended to be a full-stop on the discussion and conclusive statement that yawns aren't contagious (or are with an exact factor of 2.65) - merely that there are assumptions in the literature that need reconsidering, and a formal re-examining using alternative means (presented herein) is one appropriate way to proceed.

In examining the ultimate role yawns play, this paper has gone some way in answering important questions. To what extent is Type-1 error a problem? It is nontrival. Is there a causal relationship between a trigger and a contagious yawn (more than can be inferred by ANOVAs)? Maybe; if so, a single observed yawn raises the likelihood of yawning by a factor of about 2.65. Can this tool be applied to future yawn research? Absolutely. How seriously have we examined the null hypothesis? Not seriously enough. Are there a variety of unasked questions which are fundamental in allowing evolutionary hypothesizing? YES!

In designing a formal model of yawn contagion several important questions arose which have not been sufficiently addressed in the literature, and which have essentially been buried by common methodologies: Is the observation of one trigger yawn differentially more or less influential than the observation of two or more trigger yawns in the same period? Given the current manner in which yawns are aroused by way of 
minutes of continuous yawn stimuli, this needs to addressed. To what extent does contagion diminish over time, and what shape does the trace decay over time? Present assumptions about 3- and 5-min windows assume a flat function that is equally contagious at each minute after a trigger, but which returns to 0 after an arbitrary (and a-theoretical) threshold. The present data and model (as well as improved accountability in the future) might better illustrate in what manner yawns are contagious over time. Why is it so hard to observe a contagious yawn in the wild? Because they don't happen that often, and the increase in likelihood above the base-rate is quite modest (so modest that the observation of a true effect in the wild is likely below our threshold for being able to detect an effect of the given magnitude). These, and other questions, need to be answered to improve our understanding of what it means to say 'yawns are contagious'.

In attempting to resolve and quantify some of these questions, we can now ask more important questions (that can hopefully resolve current 'are for'-focused debates). To the extent that yawns allow evolutionary inference, we can now compare not just their role, but the magnitude of their influence, to other contagious behaviours. To the extent that yawns are contagious and informative, are they more so than, say, contagious itching (Feneran et al. 2013; Schut et al. 2015)? Contagious itching shares many features with contagious yawning (e.g., deep evolutionary history, associations with environmental factors, personal history and experience, social-cognitive factors, and illness; Schut et al. 2015). Indeed it is possible that itching is more contagious than yawning, and the data presented herein goes halfway to resolving that comparison. Likewise for whether or not laughter (Provine 2004) or smiles (Dimber et al. 2000; Surakka and Hietanen 1998), or any other social- and socially-informed behaviour is more contagious and informative than yawning. Inasmuch as it makes sense to suggest that yawns are a mechanism for facilitating social synchronization (as one example), does it make sense to say also that itching serves a similar role? Only better resolution of these questions (and more clearly quantified comparisons to other contagious / releasing behaviours) can inform our understanding.

There are several other approaches that can address issues in this literature. As was the case with this study, pre-registration can go some distance to enhancing experimental transparency, reducing experimenter degrees of freedom, and forking-paths decisions, and will not only influence what is measured and reported, but what is published. Second, given the historical precedent that yawning is contagious (dating back to antiquity) it may be reasonable to infer that the belief that yawns are contagious (perhaps erroneously arrived at) has spread to such an extent the belief itself - that yawns are contagious (widely, but not universally held) - accounts for considerable variance in whether or not yawns are spread within a group (thereafter maintained by pattern recognition error and confirmation bias). Measuring this as a variable, and potentially undertaking significant cross-cultural research to establish that evolutionary theorizing is appropriate, is necessary for advancing the field.

To conclude we make a singular claim: It is time to take more seriously the null hypothesis (and all this implies) and attempt to falsify our claims. Our own subjective experience and confidence that yawns are contagious needs to be put aside so that our methods of induction and attributions of contagious yawns can be appraised for their appropriateness. Management of type- 1 error must be resolved. Other tools for examining the phenomenon must now be considered in determining how best to answer the 
kinds of questions we're asking. To that end we make our model publicly available with instructions, and offer our services in extending the model to account for wide range of documented correlates and factors associated with contagious yawning. We hope this will underpin ongoing discussion and resolution of longstanding debates.

Acknowledgements This project began on the 12th of October 2015. We would like to acknowledge the undergrads who graciously endured being bored for 50 uninterrupted minutes. Further, we would like to thank both reviewers who provided valuable feedback that improved the quality of this manuscript.

Open Access This article is distributed under the terms of the Creative Commons Attribution 4.0 International License (http://creativecommons.org/licenses/by/4.0/), which permits unrestricted use, distribution, and reproduction in any medium, provided you give appropriate credit to the original author(s) and the source, provide a link to the Creative Commons license, and indicate if changes were made.

\section{References}

Anderson, J., \& Meno, P. (2010). Psychological influences on yawning in children. Current Psychology Letters, 26(1), 1-9.

Armstrong, E. A. (1951). The nature and function of animal mimesis. Bulletin of Animal Behavior, 10, 46-49.

Baenninger, R., \& Greco, M. (1991). Some antecedents and consequences of yawning. The Psychological Record, 41(1980), 453-460.

Bartholomew, A. J., \& Cirulli, E. T. (2014). Individual variation in contagious yawning susceptibility is highly stable and largely unexplained by empathy or other known factors. PloS One, 9(3), e91773. doi:10.1371 /journal.pone.0091773.

Bonabeau, E. (2002). Agent-based modeling: methods and techniques for simulating human systems. Proceedings of the National Academy of Sciences of the United States of America, 99(Suppl 3), 72807287. doi:10.1073/pnas.082080899.

Burns, B., \& Corpus, B. (2004). Randomness and inductions from streaks: "Gamble's fallacy" versus "hot hand". Psychonomic Bulletin \& Review, 11(1), 179-184.

Daquin, G., Micallef, J., \& Blin, O. (2001). Yawning. Sleep Medicine Reviews, 5(4), 299-312.

Demuru, E., \& Palagi, E. (2012). In bonobos yawn contagion is higher among kin and friends. PloS One, 7(11), e49613. doi:10.1371/journal.pone.0049613.

Dimber, U., Thunberg, M., \& Elmehed, K. (2000). Unconscious facial reactions to emotional facial expressions. Psychological Science, 11(1), 86-89.

Eibl-Eibesfeldt, I. (1975). Ethology, the Biology of Behavior ((Vol 2nd ed.). New York: Holt, Rinehart and Winston.

Feneran, A. N., O'Donnell, R., Press, A., Yosipovitch, G., Cline, M., Dugan, G., et al. (2013). Monkey see, monkey do: contagious itch in nonhuman primates. Acta Dermato-Venereologica, 93(1), $27-29$. doi:10.2340/00015555-1406.

Gallup, A. (2011). Why do we yawn? Primitive versus derived features. Neuroscience and Biobehavioral Reviews, 35(3), 765-769. doi:10.1016/j.neubiorev.2010.09.009.

Gallup, A., \& Gallup, J., G. G. (2008). Yawning and thermoregulation. Physiology and Behavior, 95, 10-16.

Gallup, A., Swartwood, L., Militello, J., \& Sackett, S. (2015). Experimental evidence of contagious yawning in budgerigars (Melopsittacus undulatus). Animal Cognition, 18(5), 1051-1058. doi:10.1007/s10071-015-0873-1.

Gallup, A., Church, A. M., Miller, H., Risko, E. F., \& Kingstone, A. (2016). Social presence diminishes contagious yawning in the laboratory. Scientific Reports, 6, 25045. doi:10.1038/srep25045.

Gardner, M. (1970). The fantastic combinations of John Conway's new solitaire game "life". Scientific American, 223(October), 120-123.

Gelman, A., \& Loken, E. (2013). The garden of forking paths: why multiple comparisons can be a problem, even when there is no "fishing expedition" or "p-hacking" and the research hypothesis was posited ahead of time. Department of Statistics: Coumbia University.

Gilovich, T., Vallone, R., \& Tversky, A. (1985). The hot hand in basketball: on the misperception of random sequences. Cognitive Psychology, 17, 295-314.

Grimm, V., Berger, U., Bastiansen, F., Eliassen, S., Ginot, V., Giske, J., et al. (2006). A standard protocol for describing individual-based and agent-based models. Ecological Modelling, 198(1-2), 115-126. doi:10.1016/j.ecolmodel.2006.04.023. 
Guggisberg, A. G., Mathis, J., Schnider, A., \& Hess, C. W. (2010). Why do we yawn? Neuroscience and Biobehavioral Reviews, 34(8), 1267-1276. doi:10.1016/j.neubiorev.2010.03.008.

Guggisberg, A. G., Mathis, J., Schnider, A., \& Hess, C. W. (2011). Why do we yawn? The importance of evidence for specific yawn-induced effects. Neuroscience and Biobehavioral Reviews, 35(5), 1302-1304. doi:10.1016/j.neubiorev.2010.12.004.

Madsen, E. A., Persson, T., Sayehli, S., Lenninger, S., \& Sonesson, G. (2013). Chimpanzees show a developmental increase in susceptibility to contagious yawning: a test of the effect of ontogeny and emotional closeness on yawn contagion. PloS One, 8(10), e76266. doi:10.1371/journal.pone.0076266.

Marewski, J., \& Olsson, H. (2009). Beyond the null ritual. Zeitschrift für Psychologie / Journal of Psychology, $217(1), 49-60$.

Massen, J. J., Church, A. M., \& Gallup, A. C. (2015). Auditory contagious yawning in humans: an investigation into affiliation and status effects. Frontiers in Psychology, 6, 1735. doi:10.3389 /fpsyg.2015.01735.

Norscia, I., \& Palagi, E. (2011). Yawn contagion and empathy in Homo sapiens. PloS One, 6(12), e28472. doi:10.1371/journal.pone.0028472.

Norscia, I., Demuru, E., \& Palagi, E. (2016). She more than he: gender bias supports the empathic nature of yawn contagion in Homo sapiens. R Soc Open Sci, 3(2), 150459. doi:10.1098/rsos.150459.

O'Hara, S. J., \& Reeve, A. V. (2011). A test of the yawning contagion and emotional connectedness hypothesis in dogs, Canis familiaris. Animal Behaviour, 81(1), 335-340. doi:10.1016/j. anbehav.2010.11.005.

Palagi, E., Leone, A., Mancini, G., \& Ferrari, P. F. (2009). Contagious yawning in gelada baboons as a possible expression of empathy. Proceedings of the National Academy of Sciences of the United States of America, 106(46), 19262-19267. doi:10.1073/pnas.0910891106.

Platek, S. M., Critton, S. R., Myers, T. E., \& Gallup, G. G. (2003). Contagious yawning: the role of self-awareness and mental state attribution. Cognitive Brain Research, 17(2), 223-227. doi:10.1016/s0926-6410(03)00109-5.

Provine, R. R. (1986). Yawning as a stereotyped action pattern and releasing stimulus. Ethology, 72(2), 109122.

Provine, R. R. (1989). Faces as releasers of contagious yawning- an approach to face detection using normal human subjects. Bulletin of the Psychonomic Society, 27(3), 211-214.

Provine, R. R. (2004). Laughing, tickling, and the evolution of speech and self. Current Directions in Psychological Science, 13(6), 215-218. doi:10.1111/j.0963-7214.2004.00311.x.

Provine, R. R., \& Hamernik, H. (1986). Yawning: effects of stimulus interest. Bulletin of the Psychonomic Society, 24(6), 437-438.

Romero, T., Ito, M., Saito, A., \& Hasegawa, T. (2014). Social modulation of contagious yawning in wolves. PloS One, 9(8), 1-6. doi:10.1371/journal.pone.

Rundle, B. K., Vaughn, V. R., \& Stanford, M. S. (2015). Contagious yawning and psychopathy. Personality and Individual Differences, 86, 33-37. doi:10.1016/j.paid.2015.05.025.

Schut, C., Grossman, S., Gieler, U., Kupfer, J., \& Yosipovitch, G. (2015). Contagious itch: what we know and what we would like to know. Frontiers in Human Neuroscience, 9, 57. doi:10.3389/fnhum.2015.00057.

Seki, Y., Nakatani, Y., Kita, I., Sato-Suzuki, I., Oguri, M., \& Arita, H. (2003). Light induces cortical activation and yawning in rats. Behavioural Brain Research, 14, 65-73.

Shoup-Knox, M., Gallup, A. C., Gallup, J., \& McNay, E. (2010). Yawning and stretching predict brain temperature chagnes in rats: support for the thermoregulatory hypothesis. Frontiers in Evolutionary Neuroscience, 2, 1-5.

Simonsohn, U., Nelson, L., \& Simmons, J. P. (2014a). P-curve and effect size: Correcing for Publicaiton bias using only significant results. Perspectives on Psychological Science, 9(6), 666-681.

Simonsohn, U., Nelson, L., \& Simmons, J. P. (2014b). Supplemental material for P-curve: a key to the filedrawer. Journal of Experimental Psychology: General. doi:10.1037/a0033242.supp.

Surakka, V., \& Hietanen, J. (1998). Facial and emotional reactions to Duchenne and non-Duchenne smiles. International Journal of Psychophysiology, 29(1), 23-33.

Thorpe, W. H. (1963). Learning and instinct in animals (( Vol 2nd ed.). Cambridge: Harvard University Press.

Walusinkski, O. (2009). Yawning in diseases. European Neurology, 62, 180-187.

Yoon, J. M. D., \& Tennie, C. (2010). Contagious yawning: a reflection of empathy, mimicry, or contagion? Animal Behaviour, 79(5), e1-e3. doi:10.1016/j.anbehav.2010.02.011.

Zentall, T. R. (2001). Imitation in animals: evidence, function, and mechanisms. Cybernetics and Systems, 32(1-2), 53-96. doi:10.1080/019697201300001812.

Zilli, I., Giganti, F., \& Salzarulo, P. (2007). Yawning in morning and evening types. Physiology \& Behavior, 91(2-3), 218-222. doi:10.1016/j.physbeh.2007.02.015.

Zimara, M. (1580). Aristotelis ac philsophorum medicorumque compendium. Venice: Farreus. 